\title{
Erratum: Pulsar Timing Array Based Search for Supermassive Black Hole Binaries in the Square Kilometer Array Era [Phys. Rev. Lett. 118, 151104 (2017)]
}

\author{
Yan Wang $\odot$ and Soumya D. Mohanty®
}

(Received 2 April 2020; published 22 April 2020)

DOI: 10.1103/PhysRevLett.124.169901

We found errors in Eqs. (6) and (7). The correct equations read

$$
\begin{gathered}
\left(\frac{\rho}{30}\right)=\kappa \mathcal{G}(\alpha, \delta)\left(\frac{\zeta}{5.1 \times 10^{-9}}\right) \\
\left(\frac{\rho}{30}\right)=\kappa \mathcal{G}(\alpha, \delta)\left(\frac{h}{6.4 \times 10^{-16}}\right)\left(\frac{f_{\mathrm{gw}}}{2 \times 10^{-8} \mathrm{~Hz}}\right)^{-1}
\end{gathered}
$$

These equations affect only the parts of the Letter where a conversion was required from the signal to noise ratio of a gravitational wave signal to the corresponding strain. The resulting changes, listed below, are confined to the numbers in the four paragraphs following Eqs. (6) and (7). A small subset of these numbers also appear in the abstract of the Letter and require corresponding changes.

(1) Binary systems detectable in a given redshift range now have larger chirp masses: (i) The redshifted chirp mass of a system that will be visible in the redshift range $[0.95,1.55]$ changes from $10^{9} M_{\odot}$ to $4 \times 10^{9} M_{\odot}$. (ii) The redshifted chirp mass of a system that will be visible out to $z=28.03$ changes from $10^{10} M_{\odot}$ to $4 \times 10^{10} M_{\odot}$. Abstract: The rest-frame chirp mass corresponding to the new redshifted chirp mass is $1.36 \times 10^{9} M_{\odot}$ instead of $3.4 \times 10^{8} M_{\odot}$.

(2) If $\rho=30$ is used as a detection threshold, a nondetection can rule out a GW strain of $\geq 5.2 \times 10^{-16}$ at $f_{\mathrm{gw}}=2 \times 10^{-8} \mathrm{~Hz}$. As mentioned in the abstract, the sky averaged upper limit on strain amplitude will be improved by about 2 orders of magnitude rather than about 3 orders of magnitude over existing limits.

(3) A nondetection of the GW signal at $\rho \geq 30$ from PG 1302-102 with a SKA era PTA will rule out, with very high confidence, a value of $\mathcal{M}_{c} \geq 10^{9.27} M_{\odot}$ (rather than $10^{8.67} M_{\odot}$ ). The corresponding upper limit on the rest frame total mass is $\leq 10^{9.52} M_{\odot}$ (rather than $10^{9.01} M_{\odot}$ ), assuming equal mass components. For PSO J334 + 01, the signal will have $\rho \gtrsim 16$ (rather than $\rho>100$ ) regardless of the uncertainty in the chirp mass. This is still a strong signal that can be detected with high confidence.

Results in the Letter that were based on the signal to noise ratio alone, which include all the figures, remain unchanged. Overall, there is no qualitative change in the conclusions stated in the Letter. 